\title{
The Identification of Frequency Hopping Signal Using Compressive Sensing
}

\author{
Jia YUAN ${ }^{1}$, Pengwu TIAN ${ }^{2}$, Hongyi YU \\ Department of Communication Engineering, Institute of Information Technology, Zhengzhou, Henan, China \\ Email: ${ }^{1}$ hyajia@126.com; ${ }^{2}$ tpw0802@163.com
}

\begin{abstract}
Compressive sensing (CS) creates a new framework of signal reconstruction or approximation from a smaller set of incoherent projection compared with the traditional Nyquist-rate sampling theory. Recently, it has been shown that CS can solve some signal processing problems given incoherent measurements without ever reconstructing the signals. Moreover, the number of measurements necessary for most compressive signal processing application such as detection, estimation and classification is lower than that necessary for signal reconstruction. Based on CS, this paper presents a novel identification algorithm of frequency hopping $(\mathrm{FH})$ signals. Given the hop interval, the FH signals can be identified and the hopping frequencies can be estimated with a tiny number of measurements. Simulation results demonstrate that the method is effective and efficient.
\end{abstract}

Keywords: compressive sensing, frequency hopping signal, identification

\section{Introduction}

With so many good advantages such as anti-jam, antiinterception, high security and so on, the technique of frequency hopping spread spectrum (FHSS) has been extensively applied in many areas, especially in military domain. The detection and interception of FH signals can be addressed in several methods of which wide band or channelized receiver, time-frequency distribution, and cyclostationary processing are typical ones [1-4]. For all the methods above, the extremely large requirement of measurements is one of the most serious disadvantages, which can be a bottleneck in the application of identification of high speed wide band FH signals. Recently, there have been some active attempts on signal processing with the advantage of CS for the sparse or compressive signals [5-8]. However, most of them are limited within the area of statistical inference tasks which need the prior knowledge of the probability density distribution of signals. Besides, it is seldom to be studied on how to develop the potential of CS to make processing of $\mathrm{FH}$ signal which is one of the most important sparse or compressive signals.

This paper makes use of the sparsity of FH signals on the local Fourier basis, and then presents a novel identification algorithm of $\mathrm{FH}$ signals with the compressive measurements. Given the hop interval, the FH signals can be identified and the hopping frequencies can be estimated without reconstructing the signals.

\section{Compressive Sensing Background}

\subsection{Representation and Sparsity of Signal}

Nyquist-rate sampling is the classical method to describe a signal with its bandlimitedness, while CS aims to completely describe a signal with its sparsity or compressibility to reduce the required number of measurements [9].

A signal $\mathrm{X}$ can be viewed as an $N \times 1$ column vector in $\mathbb{R}^{N}$ with elements $x[n], n=1,2, \ldots, N$. Let the matrix $\Psi=\left[\psi_{1}, \psi_{2}, \ldots \psi_{N}\right]$ have columns which form a basis of vectors in $\mathbb{R}^{N}$. And then, any signal $\mathrm{x}$ can be expressed as:

$$
\mathrm{x}=\sum_{i=1}^{N} s_{i} \psi_{i} \quad \text { or } \quad \mathrm{x}=\Psi \mathrm{s}
$$

where $\mathrm{S}$ is the $N \times 1$ column vector of weighting coefficients $s_{i}=\left\langle\mathrm{x}, \psi_{i}\right\rangle$.

When we say that $\mathrm{x}$ is $K$-sparse, we mean that it is well reconstructed or approximated by a linear combination of just $K$ basis vectors from $\Psi$, with $K \ll N$. That is, there are only $K$ of the $s_{i}$ in (1) are nonzero and $(N-K)$ are zero.

\subsection{Incoherent Measurements}

Consider a generalized linear measurement process of a signal $\mathrm{x}$ which is $K$-sparse. Let $\Phi$ be an $M \times N$ measurement matrix, $M \ll N$ where the rows of $\Phi$ 
are incoherent with the columns of $\Psi$. The incoherent measurements can be obtained by computing $M$ inner products between $\mathrm{x}$ and the rows of $\Phi$ as in $y_{j}=\left\langle x, \phi_{j}\right\rangle$. It can also be expressed as:

$$
\mathrm{y}=\Phi \mathrm{x}=\Phi \Psi \mathrm{s}=\Theta \mathrm{s}
$$

where $\Theta:=\Phi \Psi$ is an $M \times N$ matrix. It is proved that $\Phi$ dose not depend on the signal $\mathrm{x}$ and it can be constructed as a random matrix such as Gaussian matrix. And the CS theory shows that there is an over-measuring factor $c>1$ such that only $M:=c K$ incoherent measurements are required to reconstruct $\mathrm{x}$ with high probability [9-11]. That is, only $c K$ incoherent measurements include all of the salient information in the $K$-sparse signal $\mathrm{x}$, which provides the theory support on the signal processing only given the incoherent measurements without reconstructing the signals.

\subsection{Reconstruction}

With the salient information included in the incoherent measurements, there have been several kinds of reconstruction algorithms including $l_{1}$ minimization, greedy algorithm, matching pursuit and so on [12-15]. Since this paper is concentrated on FH signal identification without signal reconstruction, we don't discuss reconstruction algorithms in detail here.

\section{Compressive Identification for FH Signal}

With the good sparsity of FH signals on the local Fourier basis, we now show that incoherent measurements can be used to solve the identification problem without ever reconstructing the signal. In this process, it is able to save significantly on the number of measurements required.

\subsection{Compressive Identification Problem Setup}

FH signals are sparse in a time-frequency representation as short-time Fourier transform, and they are always wideband when there is no prior restriction on the frequencies of the local sinusoid [16]. Therefore, the measurements obtained with the traditional Nyquist-rate sampling could be excessive and hard to meet with the present ability of hardware instrument.

Now, consider a FH signal which consists of a sequence of windowed sinusoids with frequencies distributed between $f_{1}$ and $f_{2} \mathrm{~Hz}$. The bandwidth of this signal is $B=f_{2}-f_{1} \mathrm{~Hz}$, which asks for sampling above the Nyquist rate of $2\left(f_{2}-f_{1}\right) \mathrm{Hz}$ to avoid aliasing. However, the expression of the signal at any single hop is extremely simple: it consists of only one sinusoid of which bandwidth is extremely less than $B$ [16]. Hence, CS could make identi-

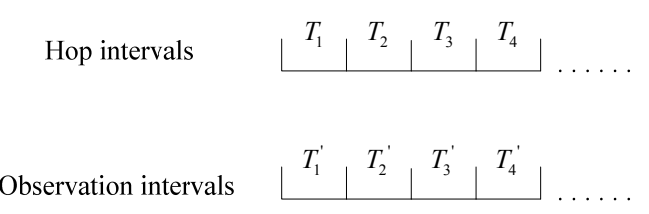

Figure 1. Hop intervals and observation intervals in the condition of 1-sparse

fication of FH signals possible with a sampling rate that is extremely less than the Nyquist rate.

Let the observation interval equal to the hop interval. If the start of the FH signal can be captured exactly, the signal can be observed synchronously as depicted in Figure 1 and it has 1-sparse representation on the local Fourier basis within each of hop interval. Otherwise, as depicted in Figure 2, the signal within each of hop interval will have 2-sparse representation since only two of the hopping frequencies appear in every single observation interval.

We observe $\mathrm{y}=\Phi_{\mathrm{x}}$ instead of $\mathrm{x}$ and our goal is to identify the FH signal and estimate its hopping frequencies with $y$ and its connection with $\Theta$.

\subsection{1-Sparse Compressive Identification}

The amplitudes of Fourier coefficients of some FH signal within an observation interval have been shown in Figure 3 which dedicates that all the coefficients are almost zero except for only one single large coefficient.

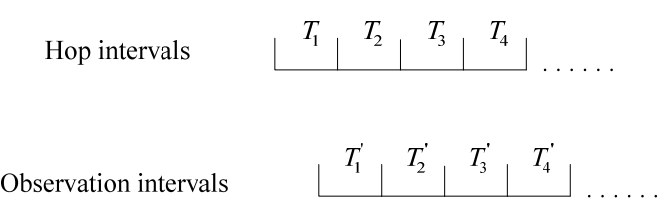

Figure 2. Hop intervals and observation intervals in the condition of 2-sparse

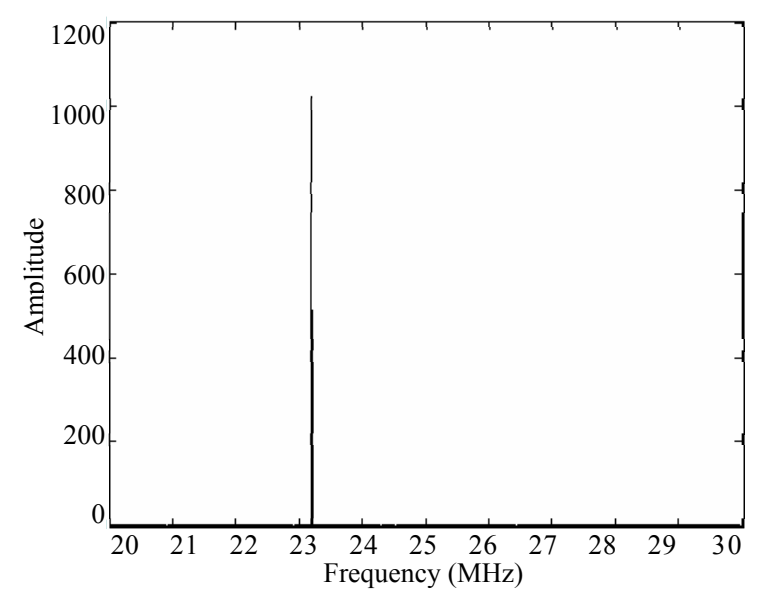

Figure 3. The amplitudes of Fourier coefficients of some FH signal within an observation interval in the condition of 1-sparse 


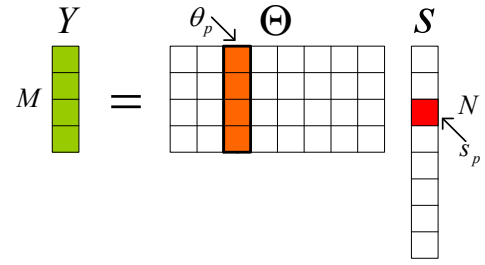

Figure 4. Measurement process in the condition of 1-sparse

The process of 1-sparse compressive measurement is depicted in Figure 4. We aim to find the position of nonzero $s_{p}$ indicating the hopping frequency of a particular interval.

Since $\mathrm{y}$ is obtained by multiplying the nonzero $s_{p}$ by its corresponding column vector $\theta_{p}$, the hopping frequency can be estimated given y and $\Theta$. A direct method to estimate the position of nonzero $s_{p}$ is to search for the position of $\theta_{p}$ which can be decided by calculating the angles between y and each column vector of $\Theta$ in the vector space as only the angle between y and $\theta_{p}$ is zero in the ideal condition. Since $\Theta$ is also a random Gaussian matrix if $\Phi$ is chosen to be a random Gaussian matrix, the angle between y and another column vector of $\Theta$ is also zero with extremely low probability. Taking account of the effect of noise, we design the estimation algorithm of hopping frequency as follows:

1) Obtain the incoherent measurements y with $\Phi$.

2) Calculate the cosine of angles between $y$ and each column vector $\theta_{i}$ in the vector space

$$
\cos \left(\theta_{i}, \mathrm{y}\right)=\frac{\theta_{i}^{H} \mathrm{y}}{\left\|\theta_{i}\right\|_{2}\|\mathrm{y}\|_{2}}
$$

where $H$ denotes conjugate transpose.

3) Select the column vector that maximizes $\cos \left(\theta_{i}, y\right)$, and define the position of this vector as estimation of hopping frequency

$$
\hat{f}=\arg \max _{i} \cos \left(\theta_{i}, \mathrm{y}\right)
$$

After several intervals of observation and estimation of hopping frequencies, the time-frequency curve of the signal can be obtained and the FH signal has been identified in the condition of 1-sparse.

\subsection{Sparse Compressive Identification}

Different from the condition of 1-sparse, Figure 5 shows that there are two large coefficients within an observation interval as each observation interval covers parts of two hop intervals in the condition of 2-sparse depicted in Figure 2.

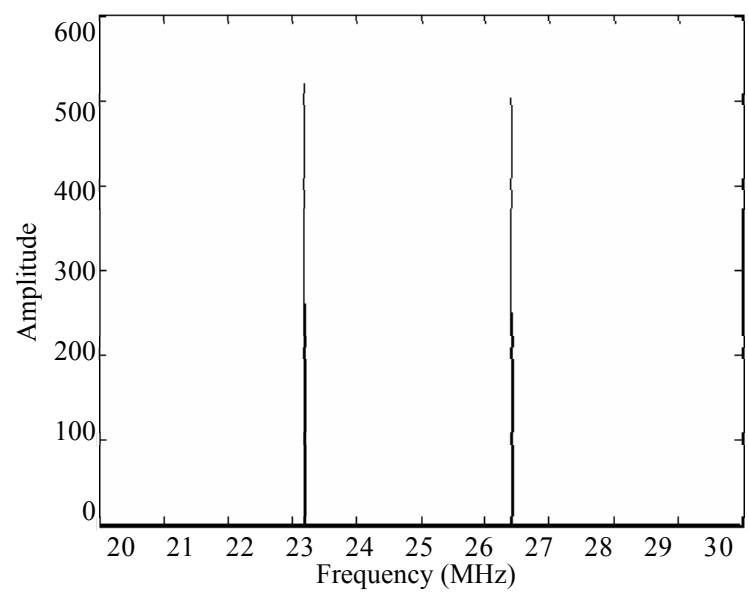

Figure 5. The amplitudes of Fourier coefficients of some FH signal within an observation interval in the condition of 2-sparse

The process of 2-sparse compressive measurement is shown is Figure 3 which dedicates that $\mathrm{y}$ is a linear combination of two column vectors $\theta_{p 1}$ and $\theta_{p 2}$ corresponding to the two nonzero coefficients $s_{p 1}$ and $s_{p 2}$ indicating the two hopping frequencies within a particular observation interval. And y is also a linear combination of another two column vectors of $\Theta$ with extremely low probability, since $\Theta$ is a random Gaussian matrix.

Therefore, the two hopping frequencies can be estimated by deciding the subspace comprised of $\theta_{p 1}$ and $\theta_{p 2}$ in $\Theta$. The estimation algorithm is as follows:

1) Obtain the incoherent measurements y with $\Phi$.

2) Calculate the orthogonal projection of y $P_{y}$ onto the subspace $L_{i j}$ comprised of any two column vectors $\theta_{i}$ and $\theta_{j}$,

$$
P_{y}=P_{L_{i j}}^{\perp} \mathrm{y}
$$

where $P_{L_{i j}}^{\perp}$ is orthogonal projector expressed by:

$$
P_{L_{i j}}^{\perp}=V\left(V^{H} V\right)^{-1} V^{H}
$$

where $V=\left[\theta_{i}, \theta_{j}\right]$.

3) Select the two column vectors that maximize $P_{y}$ onto the corresponding subspace, and define the positions of these two vectors as estimation of the two hopping frequencies

$$
\left[\hat{f}_{1}, \hat{f}_{2}\right]=\arg \max _{i, j}\left(P_{\mathrm{y}}\right)
$$

Taking account of the repetition of hopping frequencies within two consecutive observation intervals in the condition of 2-sparse, we can use the estimation results 


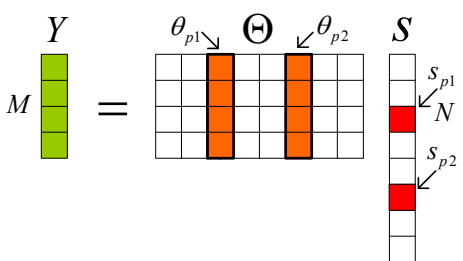

Figure 6. Measurement process in the condition of 2-sparse

of the former interval in the latter one. Only in the first interval, the algorithm is a kind of two-dimensional search as two column vectors have to be selected meanwhile. And in the successive intervals, it can be executed as a one-dimensional search (twice) as one column vector can be confirmed in according to the position information of two selected vectors of the former interval. This iterative processing can effectively reduce the computation, but obviously the error propagation can also be introduced. To solve this problem, an updating window is designed to separate the whole observation time into several segments of intervals. And in the first interval of every updating window, the two-dimensional search is executed all over again.

As the condition of 1-sparse, the time-frequency curve of the FH signal can also be obtained after several observation intervals, and the signal can be identified.

\section{Simulation Results}

To demonstrate the feasibility and effectiveness of the proposed algorithm, a wideband FH signal submerged in additive Gaussian white noise (AWGN) is considered to make the simulation experiments. This FH signal has ten hopping frequencies which are distributed uniformly between $20 \mathrm{MHz}$ and $200 \mathrm{MHz}$, and the hop interval is $1 \mathrm{~ms}$, i.e. 1000 hops per second. The other main simulation parameters are as follows: 2048-point local Fourier basis is chosen to be $\Psi$, random Gaussian matrix is chosen to be $\Phi$, and the number of observation intervals is set to 2000. Each experiment is made in the condition of both 1-sparse and 2-sparse.

First, the estimation performance of hopping frequency is evaluated by normalized mean square error (NMSE) through several intervals of observation, where NMSE is expressed by

$$
N M S E=\frac{1}{N_{T^{\prime}}} \sum_{i=1}^{N_{T^{\prime}}}\left(\frac{\hat{f}_{i}-f_{i}}{f_{i}}\right)^{2}
$$

where $\hat{f}_{i}$ is the estimation of hopping frequency that expressed by $f_{i}$ in the $i$ th observation interval and $N_{T^{\prime}}$ represents the number of observation intervals which is set to 2000 here.

Figure 7 and Figure 8 show the performance curves of 1 -sparse and 2-sparse respectively.

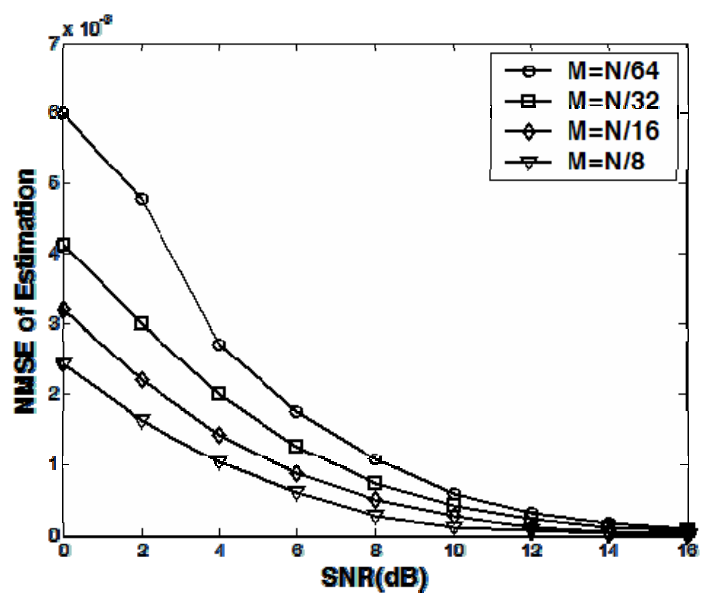

Figure 7. MSE of estimation with SNR in the condition of 1-sparse, where $\mathrm{N}=\mathbf{2 0 4 8}$ and $M$ represents the number of measurements used in this experiment experiments

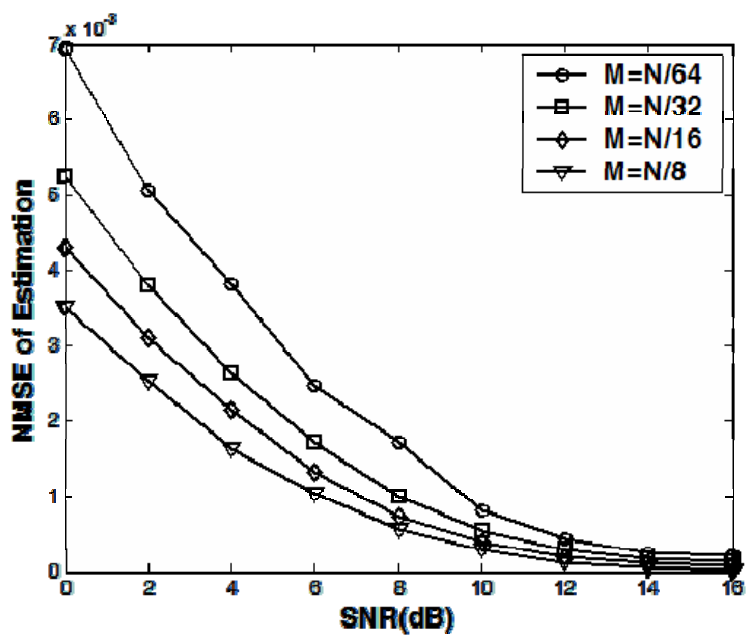

Figure 8. MSE of estimation with SNR in the condition of 2-sparse, where $\mathrm{N}=\mathbf{2 0 4 8}$ and $M$ also represents the number of measurements used in this experiment experiments. And the length of updating window is set to 40

Some conclusions can be demonstrated from Figure 7 and Figure 8. First, the hopping frequencies can be effectively estimated with a tiny number of measurements when SNR is higher than $8 \mathrm{~dB}$. Second, the performance of estimation degrades with the decrease of $M$, especially in low SNR. And finally, the performance of 1sparse is better than that of 2-sparse.

Next, the estimated time-frequency curves of the FH signal of 1-sparse and 2-sparse are depicted in Figure 9 and Figure 10 respectively when $M=N / 16$ and SNR is $10 \mathrm{~dB}$.

From the Figure 9 and Figure 10, it is shown that the estimated time-frequency curve is quite close to the real one and the FH signal can be effectively identified, especially in the condition of 1-sparse. 


\section{Conclusions}

Based on CS, this paper provides a novel method for the identification of wideband FH signal with a tiny number of incoherent measurements, which is an inspiration of real-time wideband sparse signal processing. This method can also be of great help for the detection and recognition of wideband signal in the non-cooperative communication.

There are many opportunities for future research. Identification without the information of hop interval, the picket fence effect of Fourier transformation on the performance of identification, and the theoretical bounds of $M$ with a given SNR would be discussed in the future work.

\section{REFERENCES}

[1] AYDIN L, POLYDOROS A. Hop-timing estimation for FH signals using a coarsely channelized receiver. IEEE Trans. Communication, Apr. 1996, 44(4): 516-526.

[2] ZHANG X, DU X, ZHU L. Time frequency analysis of frequency hopping signals based on Gabor spectrum method. Journal of Data Acquisition \& Processing, Jun. 2007, 22(2): 123-135.

[3] HIPPENSTIEL R, KHALIL N, FARGUES M. The use of wavelets to identify hopped signals. In 1997 Fortieth Asilomar Conf. Signals, System \& Computer, 1997, 1: 946-949.

[4] FAN H, GUO Y, XU Y. A novel algorithm of blind detection of frequency hopping signal based on second-order cyclostationarity. Proc. 2008 Image and Signal Processing Congr., 2008, 5: 399-402.

[5] HAUPT J, NOWAK R, YEH G. Compressive sampling for signal classification. In 2006 Asilomar Conf. on Signals, System \& Computer, Oct. 2006, 1430-1434.
[6] HAUPT J, NOWAK R. Compressive sampling for signal detection. Conf. Rec. 2007 IEEE Int. Conf. Acoustics Speech and Signal Processing, 2007, 3: 1509-1512.

[7] DUARTE M F, DAVENPORT M A, WAKIN M B. Multiscale random projection for compressive classification. Conf. Rec. 2007 IEEE Int. Conf. Image Processing, 2007, 6: 161-164.

[8] DUARTE M F, DAVENPORT M A, WAKIN M B, BRANIUK R G. Sparse signal detection from incoherent projection. Conf. Rec. 2006 IEEE Int. Conf. Acoustics Speech and Signal Processing, 2006, 3: 305-308.

[9] BRANIUK R. Compressed sensing. IEEE Signal Processing Magazine, Jul. 2007, 24(4): 118-121.

[10] DONOHO D. Compressed sensing. IEEE Trans. Inform. Theory, Apr. 2006, 52(4): 1289-1306.

[11] CANDES E, ROMBERG J, TAO T. Robust uncertainty principles: Exact signal reconstruction from highly incomplete frequency information. IEEE Trans. Inform. Theory, Feb. 2006 52(2): 489-509.

[12] DONOHO D, TANNER J. Sparse nonnegative solutions of underdetermined linear equations by linear programming. Proc. National Academy Science, 2005, 102(27): 9446-9451.

[13] TTOPP J A. Greed is good: Algorithmic results for sparse approximation. IEEE Trans. Inform. Theory, Oct. 2004, 50(10): 2231-2242.

[14] HAUPT J, NOWAK R. Signal reconstruction from noisy random projection. IEEE Trans. Inform. Theory, Sep. 2006, 52(9): 4036-4048.

[15] CHEN S, DONOHO D, SAUNDERS M. Atomic decomposition by basis pursuit. SIAM J. Sci. Comput., 1998, 20: 33-61.

[16] LASKA J, KIROLOS S, MASSOUD Y, BARANIUK R Random sampling for analog-to-informaion conversion of wideband signals. IEEE Dallas/CAS Workshop on Design, Application, Integration and Software, Oct. 2006, 119-122. 